\title{
Linguistic-Based Detection of Fake News in Social Media
}

\author{
Mohammad Mahyoob ${ }^{1}$, Jeehaan Algaraady ${ }^{2}$ \& Musaad Alrahaili ${ }^{1}$ \\ ${ }^{1}$ Taibah University, Madinah, Saudi Arabia \\ ${ }^{2}$ Taiz University, Taiz, Yemen \\ Correspondence: Jeehaan Algaraady, Taiz University, Taiz, Yemen. E-mail: jihan.amu@gmail.com
}

Received: September 12, $2020 \quad$ Accepted: November 1, $2020 \quad$ Online Published: November 17, 2020

doi:10.5539/ijel.v11n1p99 URL: https://doi.org/10.5539/ijel.v11n1p99

\begin{abstract}
The tremendous growth and impact of fake news as a hot research field gained the public's attention and threatened their safety in recent years. However, there is a wide range of developed fashions to detect fake contents, either those human-based approaches or machine-based approaches; both have shown inadequacy and limitations, especially those fully automatic approaches. The purpose of this analytic study of media news language is to investigate and identify the linguistic features and their contribution in analyzing data to detect, filter, and differentiate between fake and authentic news texts. This study outlines promising uses of linguistic indicators and adds a rather unconventional outlook to prior literature. It utilizes qualitative and quantitative data analysis as an analytic method to identify systematic nuances between fake and factual news in terms of detecting and comparing 16 attributes under three main linguistic features categories (lexical, grammatical, and syntactic features) assigned manually to news texts. The obtained datasets consist of publicly available right documents on the Politi-fact website and the raw (test) data set collected randomly from news posts on Facebook pages. The results show that linguistic features, especially grammatical features, help determine untrustworthy texts and demonstrate that most of the test news tends to be unreliable articles.
\end{abstract}

Keywords: fake news detection, data mining, linguistic features, text classification, content analysis, social media

\section{Introduction}

Words played a critical role in shaping the public's attitudes and opinions in news media. Recently, fake news has attracted worldwide attention and multiplied organized efforts have been dedicated to fact-checking. They attempted to counter online misinformation transmit raises in media outlets. According to Conroy (2015), Fake news detection is the projection of a news article (news report, editorial, and expose) to be intentionally deceiving. It is not a new idea, but what makes it a world attractive topic is that most people worldwide get their news from social media as it breaks the distance barriers among individuals and societies (Shu et al., 2019). On the other hand, it is the easiest, cheapest, and fastest way to publish fake news online, promoting malicious entities to create, print, and spread fake news.

In recent years, fake news for different commercial and political purposes has been emerged widely in online social networks causing real-world influences within minutes for a considerable number of users. These immense effects of fake news demand a real and robust step to identify and improve the information's trustworthiness. In the meantime, Fake news was highlighted during the 2016 U.S. presidential election campaign and became a serious threat to journalism, democracy, expression freedom, and the public's trust in governments. The chance to deceive or to be deceived becomes more and more during news production, dissemination, and consumption; thus, spotting fake content in online sources is a pressing need for social and political grounds.

As stated by Allcott and Gentzkow (2017), Fake news detection is a challenging task since it looks like real news and tricks those who do not authenticate for the reliability of the contents and sources. Moreover, the lack of available comparative information and checking news articles require careful fact-checking and evidence-collecting. In the last four decades of deception detection research has helped us learn more about how well humans can detect lies in the text. The results show that humans, to some extent, can detect deception in content but not so well at it (Conroy et al., 2015). A meta-analysis of more than 200 experiments is just $4 \%$ better than chance, as stated by Bond and DePaulo (2006). People tend to harness their cognitive efforts to change or 
hide information, which causes behavior changes and, consequently, changes in verbal and written texts. They attempt to change their writing style to fabricate individual facts for specific purposes. It contains linguistic features change, and by investigating these features, one can reveal false texts. That challenge encourages researchers to look at several fashions for detecting deceptive texts (Rao \& Rohatgi, 2000). The linguistic analysis could identify Sci.crypt anonymous authors by comparing their text contents with documents associated with the RFC database and the IPSec mailing list. Thus, the linguistic construction of news articles can help fact-checkers in identifying hoaxes and deliberate misinformation.

We can study fake news from three perspectives: (I) style: fake news writing style, (II) propagation: how fake news spread, and (III) users: how users participate in fake news and the role users can play in all these perspectives (Zafarani et al., 2019; Zhou \& Zafarani, 2018).

Hence, there is an urgent need to develop approaches for detecting fake news based on their content. In linguistic methods, the content of false texts is extracted and analyzed to relate language patterns with deception (Conroy et al., 2015). In this paper, the authors proposed a linguistic-based fake news detection method. This method empirically focuses on analyzing and investigating the news articles' linguistic characteristics in content structure and style as a foundation for news credibility inference. It attempts to differentiate between fake and real news and assess fake texts' truth value. Relying on the social and psychological theories as a systematic framework of the study, the authors attempt to examine authentic texts' explainable manual linguistic attributes and their contribution to detecting fake news. These theories stated some linguistic cues when a human being lies compared to when he or she tells the truth. Fake news tends to be less complicated to comprehend because deceivers' language style implies more straightforward sentences, fewer long sentences, and shorter words than truth-tellers (Burgoon et al., 2003). Undeutsch hypothesis states that fake statements vary in writing style and quality from factual statements (Udo Undeutsch, 1967).

Based on these attributes, this study aims to introduce qualitative and quantitative analytic research on the language of two types of news articles in the context of fake news detection. First, the authors attempt to examine and identify the real articles' linguistic features obtained from the Politi-fact site, then compared them with the linguistic features of a set of chosen news articles from Facebook to identify its trustworthiness.

The rest of this paper structure organized as follows: Section 2 represents the literature review. Section 4 introduces fake news definitions, section 3 defines data collection, and section 4 describes the study's methodology and model. Section 5 displays the results, section 6 discusses the results, and section 7 concludes the article and introduces possible future studies.

\section{Significance of the Study}

In this paper, the authors proposed a linguistic-based fake news detection method. This method focuses on analyzing and investigating the news articles' content structure and style based on the texts' linguistic characteristics to differentiate between fake and real news as a foundation for news credibility inference and assess fake texts' truth value.

Based on a set of linguistic features and attributes, this study aims to introduce qualitative and quantitative analytic research on the language of two types of news articles in the context of fake news detection. The authors compared the language of a set of news articles with the right articles obtained from politi-fact.com to identify deceptive news text's linguistic features and classify those set of news articles.

\section{Related Works}

Although Fake news detection is a hot research area, it is not a new phenomenon. Many works studied fake news in the context of their content, the way it spreads, and others its writing style (Zheng et al., 2006). Markowitz and Hancock (2014) demonstrated how linguistic patterns related to discourse dimensions could be used as cues to differentiate between fraudulent and genuine publications of the social psychologist Diederik Stapel's. Golbeck et al. (2018) utilized a word-based classification approach based on the Naive Bayes Multinomial Algorithm to identify the linguistic nuances between fake and satirical articles. Levi et al. (2019) proposed a machine learning method using semantic representation to identify fake news and satire's nuances. They used the Coh-Metrix tool for producing linguistic and discourse terms of texts and attempt to address the challenges of identifying the differences between fake news and satire. They stated that satire language seems to be more sophisticated than counterfeit articles. Newman et al. (2003) used some linguistic hints such as self-references or positive and negative words to distinguish truth-tellers from liars.

Shu et al. (2017) utilized the document's latent embedding to identify and detect false news. Wang (2017) attempted to classify fake news content based on the convolutional neural network (CNN). While Qin et al. 
(2005), in their work, attempted to explore and analyze the number of Other work has focused on analyzing the self-references, the number of words and sentences, affect, spatial and temporal information associated with deceptive content.

Ruchansky et al. (2017) stated that people widely use social media to express their feelings and emotions, and these posts can help for feature detection. They utilized social media posts to extract the differences in temporal engagement patterns between real and fake news. Burfoot and Baldwin (2009) used a support vector machine algorithm (SVM) to automatically classify the content's lexical and semantic features to differentiate between the actual and satire contents. In their works, Ott et al. (2011), Shafqat et al. (2016), Zhang and Guan (2008), Warkentin et al. (2010), Toma and Hancock (2010) tried to do an automatic detection of deceptive content. They explored different domains such as online dating, crowd founding platforms, consumer reviews websites, and online advertising.

Rubin et al. (2016) tried to detect satire news from real news using an SVM-based algorithm with five predictive features (Absurdity, Humor, Grammar, Negative Affect, and Punctuation). Their results revealed that the best prediction feature combination (Absurdity, Grammar, and Punctuation) detects satirical news with a $90 \%$ precision and $84 \%$ recall. Bessi et al. (2014) studied the spread of false news on social media. Their study proposed that users who interact using different social media are more probably use false information. Their focus was on the attention given to the false news on Facebook. Shao et al. (2016) introduced the Hoaxy platform for automatic tracking of both true and false online misinformation, relying on the efforts of other fact-checkers such as snopes.com. Zhou et al. (2019) used the theory-driven model in their proposed method for fake news early detection. This method investigates news content at different linguistic levels relying on well-established theories in social and forensic psychology.

\section{Definition of Fake News}

The term fake news is not new. It began as the news printing press started. As a term, it appeared in the Oxford Dictionary in 2017. Fake news is a fictitious article deliberately fabricated to deceive readers. It is a means to increase the amount of readership or to create psychological warfare. There are many studies about fake news, and there is no agreed definition of this term. Many studies connect fake news and other terms such as false news, rumor, misinformation, and maliciously false news. According to Allcott and Gentzkow (2017), fake news is news articles that are deliberately and verifiably false and could mislead readers. Conroy (2015) treats fake news as deceptive news, including heavy fabrication, hoaxes, and satires in his work. Balmas (2014) stated that fake news refers to satire news as they contain false content. Unlike fake news, satire news in its nature is entertainment-oriented.

\section{Methodology and Data Collection}

\subsection{Methodology}

The reliable methodology for identifying fake news is still challenging among researchers; however, some linguistic attributes are used to explore different language categories' relationships. This section introduces the methodology through which this study was processed. The researchers downloaded twenty factious articles from Politi-fact websites and twenty news articles posts on Facebook to be analyzed based on a set of linguistic characteristics. They thus assisted in classifying news texts, either true or false. Then, they clean the obtained data in the form of texts from all "stop" lists such as posters, digits, timing, and dates. They utilized the QDA tool to process the collected datasets; QDA (Qualitative Data Analysis) tool offers a data annotation with evaluating metrics for text mining. It can analyze news, survey interviews, spreadsheets, online, videos, pictures, and audio files. The analysis and detection of the collected articles' writing content structure and style based on a bundle of discriminating linguistic features and attributes are chiefly stylistic features for natural language analysis.

\subsection{Data Collection}

The first step in this study is data construction. For conducting this study, the authors obtained two datasets from social media websites as follows:

- Dataset 1: the first dataset includes 20 authentic texts download from the Politi-fact website (a fact-checking website led by Tampa Bay Times journalists to validate declares by elected officials and others on its Truth-O-Meter). The unique advantage of Politi-Fact is that every quote is rated on a 6-point scale, ranging from "True" (factual) to "Pants on-Fire False" (absurdly false).

- Dataset 2: The second dataset contains 20 news reports chosen randomly from different Facebook pages to be assessed compared to real news articles in dataset 1 . The obtained datasets are collected in the form of texts 
and processed by QDA analysis software.

\section{Linguistic Features and News Representation}

The researchers attempt to investigate and explore the differences between fake and authentic news by empirically analyzing their main linguistic characteristics for achieving promising outcomes as described below:

To detect news writing style, the authors explore and consider the absolute frequency of a bundle of linguistic attributes in news content. These attributes include "personal pronoun, proper pronoun, adverb, stative verb, to-infinitive, passive voice, reported speech, comparative adjectives, superlative adjectives, modal verbs, quotes, conjunctions, long sentences, interrogative, and negation" of which frequency obtained computationally.

These computational features across language levels represent and classify news articles on both fronts: fake and actual news. The features matrix of all examinable linguistic attributes is shown in the data analysis section below:

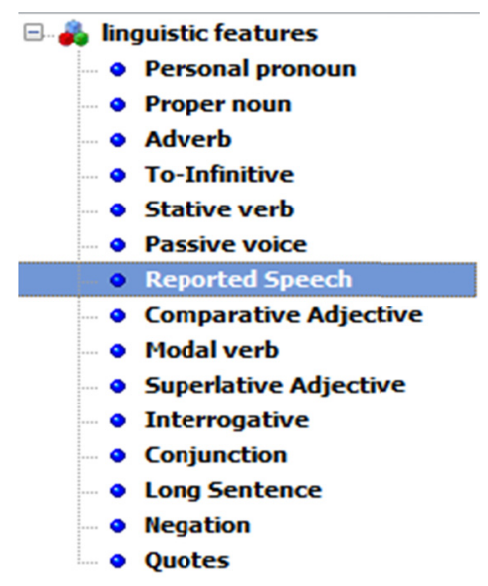

Figure 1. Linguistic features for news articles

The authors attempted to code these features as they allow performing a systematic qualitative and quantitative content analysis. Under the principle code (linguistic features), the authors conveniently assigned 16 sub-codes (linguistic attributes) manually to the relevant information in both datasets as displayed in Figure 2 below:

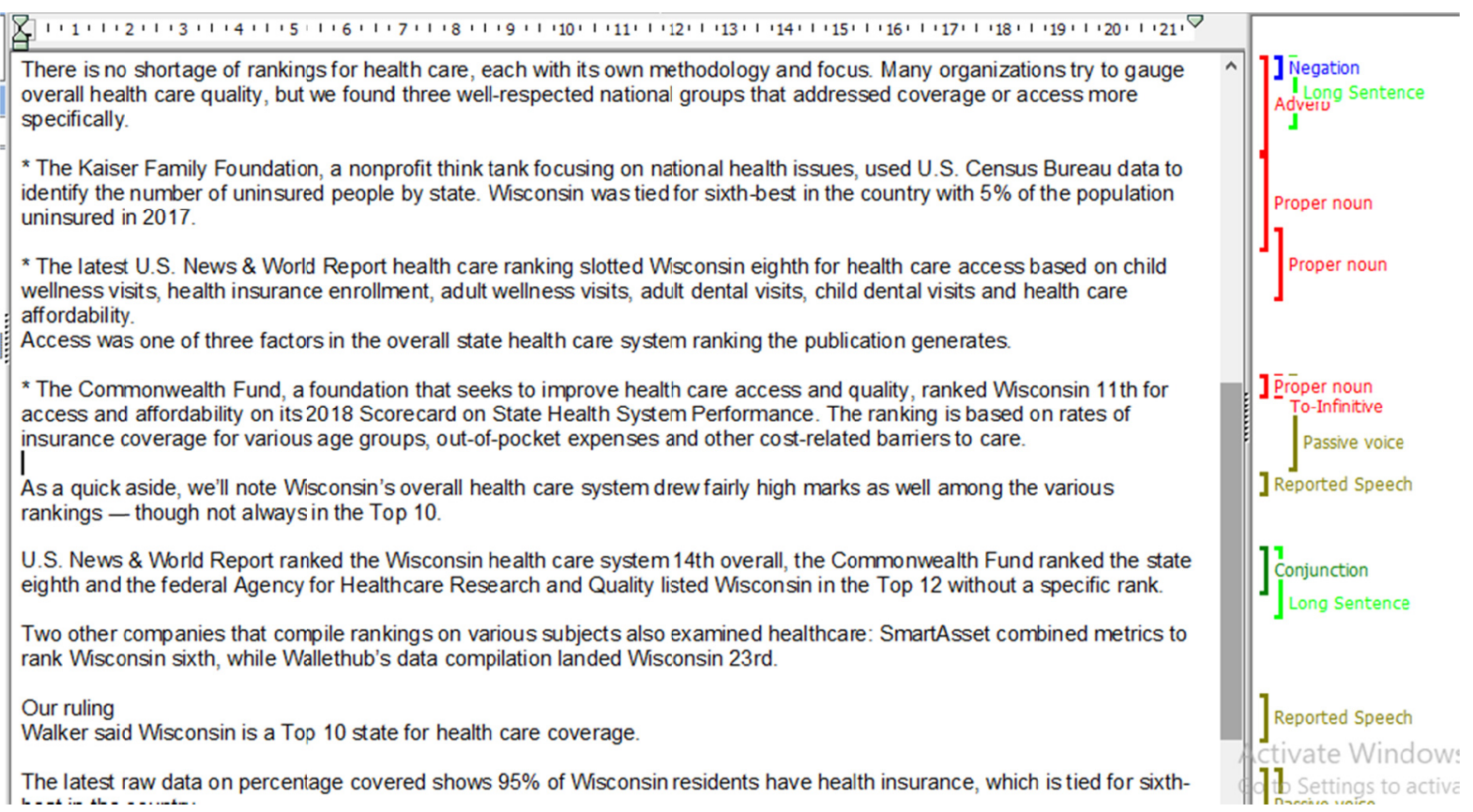

Figure 2. Sample of document codes labeling 'linguistic features and Data annotation' 
The same linguistic information was investigated in both genuine and fake texts separately. The authors utilized clustering techniques of different linguistic paradigms to extract linguistic characteristics. After tracking both types of news articles, including determining and labeling these features, the authors used the QDA tool to perform a systematic computation of the linguistic attributes' frequencies. They compared the linguistic features of true dataset 1 with the linguistic features of test dataset 2 to find the linguistic nuances. Finally, they harnessed this computational comparison to provide and identify the linguistic cues of fake news writing style that assist in classifying dataset 2. Figure 3 below illustrates the model of the study.

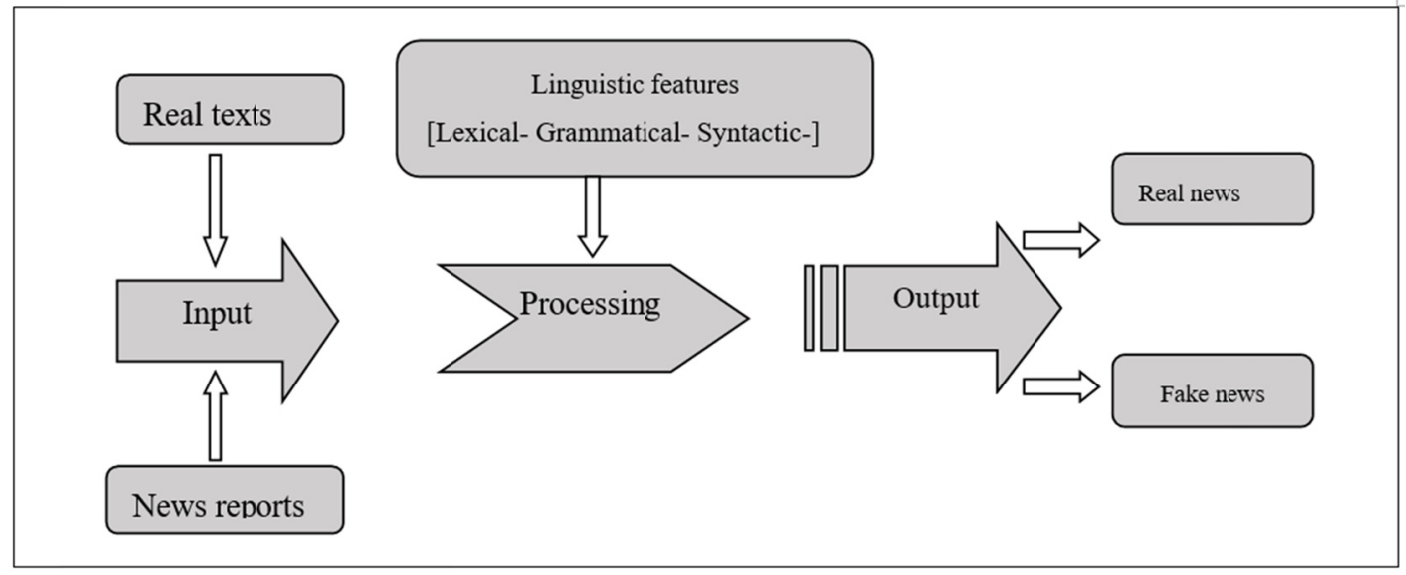

Figure 3. Model of the study

\section{Results and Findings}

This study's obtained data consists of 20 articles (authentic) collected from the Politi-fact website and 20 articles randomly chosen from different Facebook pages to assess their authenticity based on the actual reports discriminated linguistic features cues. The following graphs illustrate the application of linguistic arguments that help detect the deception in the news articles. The relation among the language elements is the core key in identifying the reasoning mode, which augments the possibility of quantitative and computational analysis of the documents.

The results present a careful sampling of the relative frequencies of codes (different features) in both datasets.

An automatic account is made for how often these features appear in the content of the obtained data. The following tabular and graphical analyses show the distribution and analysis of these features.

\subsection{Linguistic Features Distributions (LFD)}

\subsubsection{LFD for Politi-Fact Site Factious Articles}

Figure 4 shows the relative frequencies of all the tested linguistics attributes in Politi-fact site articles. It demonstrates that the four linguistic features: reported speech, passive voice, negation, and proper nouns are the top used features in these articles type. While to-infinitive, modals, and long sentences are the less used in these articles.

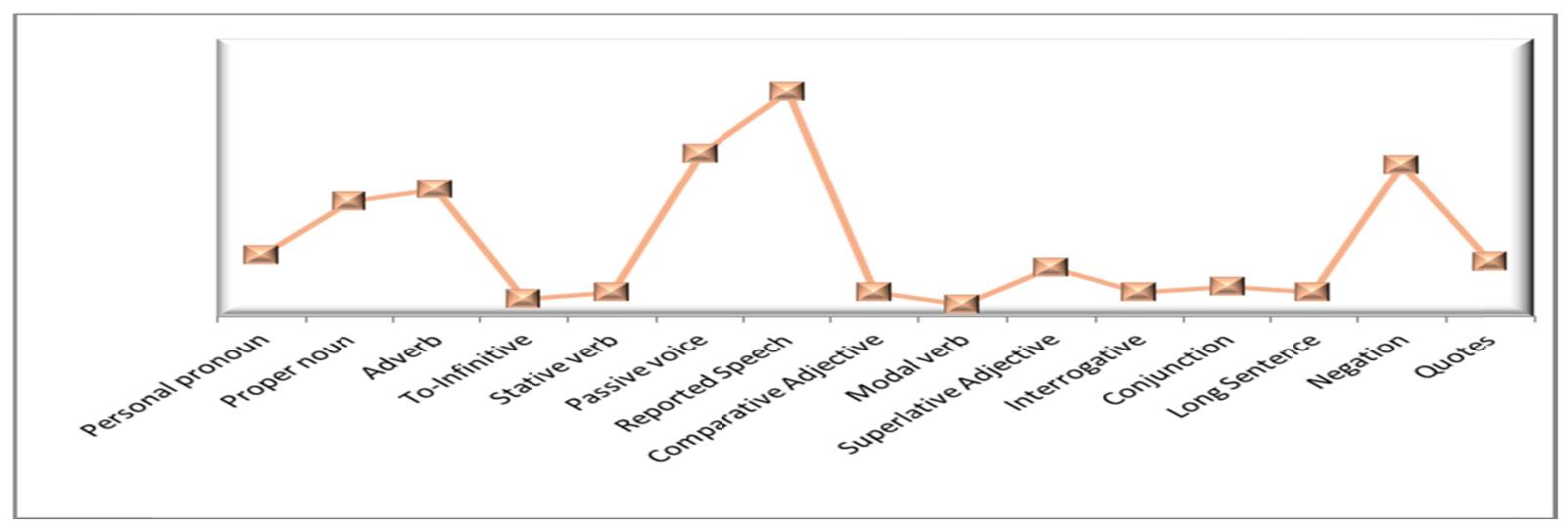

Figure 4. Linguistic features distribution for dataset 1 (Politi-fact site factious articles) 
In more detail, as observed in Table 1, a range of linguistic features can contribute to our perception of the nuances between reliable and unreliable digital news sources. Reported speech is the most frequent grammatical feature that appeared in the authentic news with a score of $19.6 \%$ and covered all news articles. Then passive voice achieved $14.7 \%$ and covered $88.9 \%$ of the collected news reports. Negation is also one of the highly used attributes in this dataset, reached a $13.0 \%$ value of frequency, and appeared in $88.9 \%$ of the articles. Trustworthy news writers tend to use adverbs, proper nouns, personal pronouns, and quotes with respective scores of $11.4 \%$, $9.2 \%, 5.4 \%$, and $4.9 \%$. Adverbs seem to cover $100 \%$ of original obtained articles, while proper nouns appeared in $88.9 \%$, personal pronouns in $55.6 \%$, and quotes in $33.3 \%$ of those articles. Superlative adjectives showed less score of $4.3 \%$ and occurred in $66.7 \%$ of dataset 1 .

Table 1. The analytic representation of linguistic feature frequencies for dataset 1

\begin{tabular}{lccccc}
\hline Code & Count & \% Codes & \% Cases & Nb Words & \% Words \\
\hline Personal pronoun & 10 & $5.50 \%$ & $55.6 \%$ & 717 & $11.1 \%$ \\
Proper noun & 17 & $9.2 \%$ & $88.9 \%$ & 812 & $12.6 \%$ \\
Adverb & 21 & $11.4 \%$ & $100.0 \%$ & 940 & $14.6 \%$ \\
Stative verb & 4 & $2.2 \%$ & $33.3 \%$ & 142 & $2.2 \%$ \\
To-Infinitive & 2 & $1.1 \%$ & $11.1 \%$ & 88 & $1.4 \%$ \\
Passive voice & 27 & $14.7 \%$ & $88.9 \%$ & 1285 & $20.0 \%$ \\
Reported Speech & 36 & $19.6 \%$ & $100.0 \%$ & 1558 & $24.2 \%$ \\
Comparative Adjective & 4 & $2.2 \%$ & $33.3 \%$ & 184 & $2.9 \%$ \\
Superlative Adjective & 8 & $4.3 \%$ & $66.7 \%$ & 398 & $6.2 \%$ \\
Modal verb & 2 & $1.1 \%$ & $22.2 \%$ & 151 & $2.3 \%$ \\
Interrogative & 4 & $2.2 \%$ & $33.3 \%$ & 103 & $1.6 \%$ \\
Conjunction & 4 & $2.2 \%$ & $22.2 \%$ & 152 & $2.4 \%$ \\
Long Sentence & 3 & $1.6 \%$ & $33.3 \%$ & 157 & $2.4 \%$ \\
Negation & 24 & $13.0 \%$ & $88.9 \%$ & 1176 & $18.3 \%$ \\
Quotes & 9 & $4.9 \%$ & $33.3 \%$ & 451 & $7.0 \%$ \\
\hline
\end{tabular}

\subsubsection{LFD for Test Articles in Dataset 2}

Figure 5 displays the relative frequencies of all the tested linguistics dataset 2 articles. As observed, reported speech, passive voice, and negation are the top three linguistic features used in dataset 2 articles while comparative, conjunctions, long sentences, and modal verbs are less used in this type of news article. As noticed, reported speech, passive voice, and negation are the top used features in both datasets 1 and 2, but the difference is in their frequencies, as shown in Tables 1 and 2.

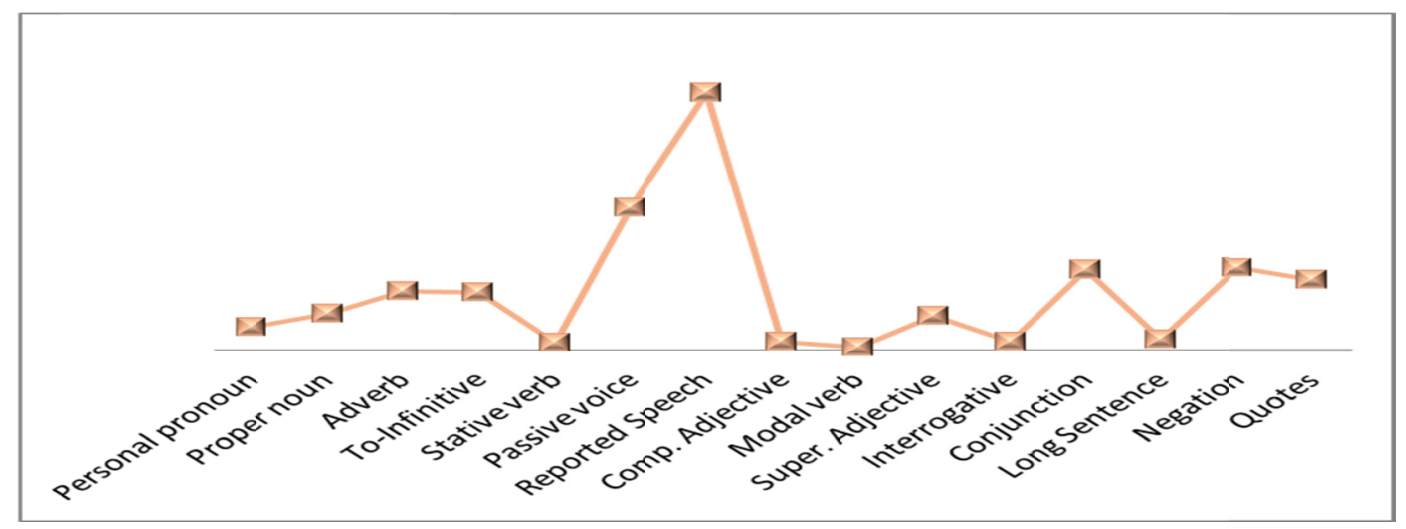

Figure 5. Linguistic features distribution for dataset 2

Table 2 displays the detailed distribution of all the chosen linguistic features within articles in dataset 2 . The proper noun was the most frequently used attribute that scored $9.1 \%$ and covered $69.2 \%$ of the collected test articles. The second most used lexical feature is an adverb with an $8.3 \%$ score and $76.9 \%$ of these articles. As observed, the reported speech attribute was excessively used in this collection, reached $24.6 \%$, and appeared in 
all the articles. It is the most utilized attribute among all the linguistic characteristics in the two obtained datasets. Passive voice is the next frequently used attribute with a $15.5 \%$ score and occurred in all articles. Negation scored $10.7 \%$, and like the attributes mentioned above, it covered $100 \%$ of the articles, i.e., all these dataset articles include negation in its several forms.

Table 2. The analytic representation of linguistic feature frequencies for dataset2.

\begin{tabular}{lccccc}
\hline Code & Count & \% Codes & \% Cases & Nb Words & \% Words \\
\hline Personal pronoun & 15 & $6.0 \%$ & $61.5 \%$ & 906 & $10.8 \%$ \\
Proper noun & 23 & $9.1 \%$ & $69.2 \%$ & 980 & $11.6 \%$ \\
Adverb & 21 & $8.3 \%$ & $76.9 \%$ & 936 & $11.1 \%$ \\
Stative verb & 6 & $2.4 \%$ & $38.5 \%$ & 204 & $2.4 \%$ \\
To-Infinitive & 5 & $2.0 \%$ & $15.4 \%$ & 136 & $1.6 \%$ \\
Passive voice & 39 & $15.5 \%$ & $92.3 \%$ & 1565 & $18.6 \%$ \\
Reported Speech & 62 & $24.6 \%$ & $100.0 \%$ & 2211 & $26.3 \%$ \\
Comparative Adjective & 4 & $1.6 \%$ & $23.1 \%$ & 184 & $2.2 \%$ \\
Superlative Adjective & 8 & $3.2 \%$ & $53.8 \%$ & 365 & $4.3 \%$ \\
Modal verb & 2 & $0.8 \%$ & $15.4 \%$ & 151 & $1.8 \%$ \\
Interrogative & 5 & $2.0 \%$ & $30.8 \%$ & 111 & $1.3 \%$ \\
Conjunction & 4 & $1.6 \%$ & $23.1 \%$ & 146 & $1.7 \%$ \\
Long Sentence & 3 & $1.2 \%$ & $23.1 \%$ & 157 & $1.9 \%$ \\
Negation & 27 & $10.7 \%$ & $92.3 \%$ & 1157 & $13.7 \%$ \\
Quotes & 17 & $6.7 \%$ & $53.8 \%$ & 736 & $8.7 \%$ \\
\hline
\end{tabular}

\subsection{Linguistic Features Coverage Within Real News Articles}

Figure 6 below explains the occurrence of all the tested linguistics features in all real news articles. It shows that the proper noun is approximately the dominant feature in most news articles, then reported speech in actual news articles.

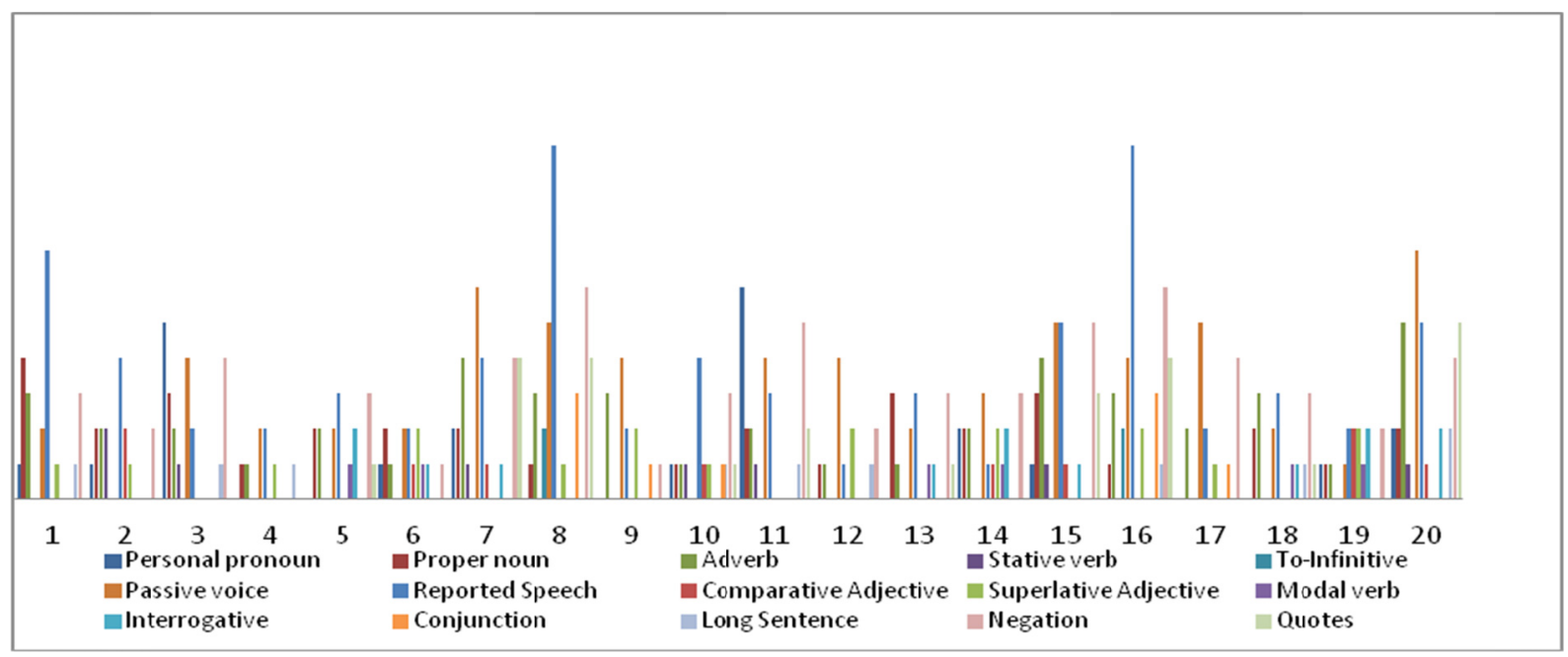

Figure 6. Graphical coverage of Linguistic features within dataset1

\subsection{Linguistic Features Coverage Within Dataset 2 News Articles}

Figure 7 displays that reported speech is the most dominant linguistic feature in approximately most analyzed datasets 2 articles, then the passive voice linguistic feature. While other top features: conjunction, negation, quotes, adverb, and proper nouns show the difference in the percentage of words and code occurrences rather than coverage within two datasets articles. 


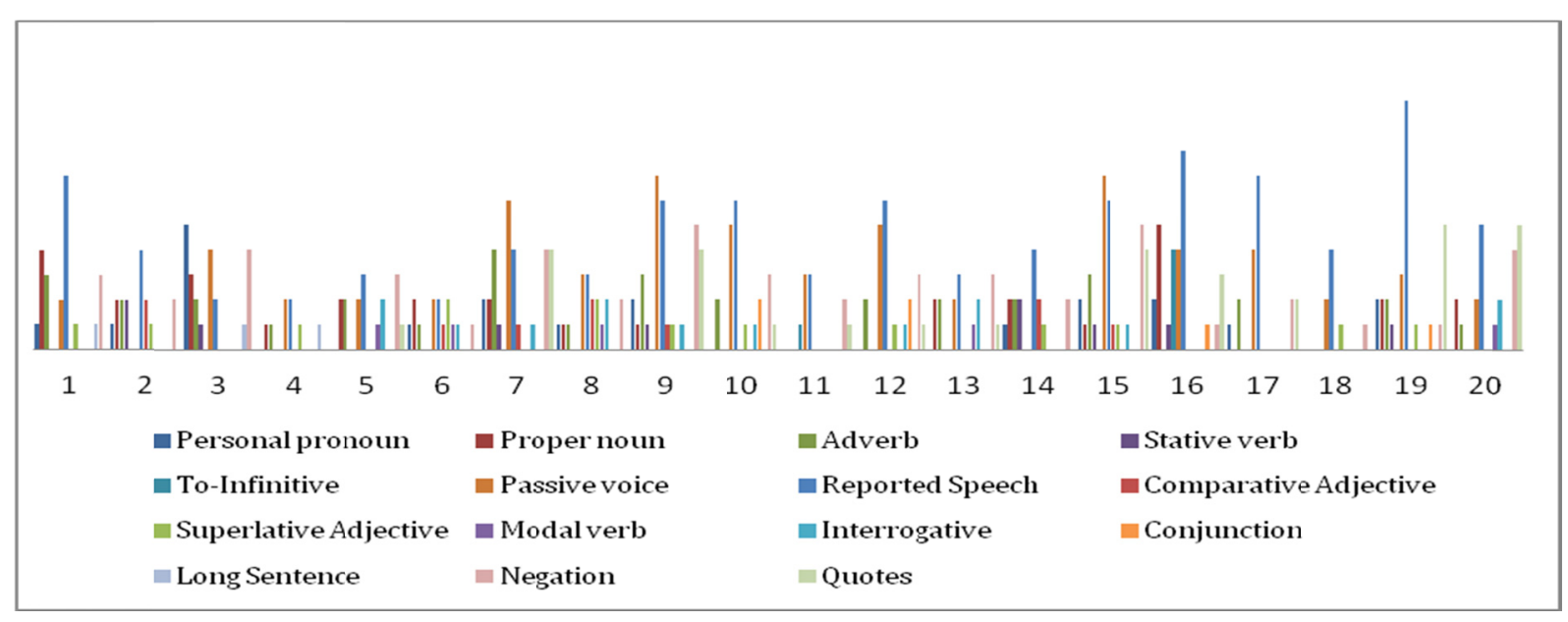

Figure 7. Graphical coverage of linguistic features within dataset 2

\section{Discussion}

This study aims to perform a qualitative and quantitative linguistic analysis of the content structure and news articles' style to identify fake news articles' linguistic features and classify news texts, either false or authentic. However, purveyors of fake news deliberately tend to be more reliable and carefully attempt to convince their audience by controlling their language and writing style to look real. Certain aspects of language "predictive deception cues" can be monitored and detected by investigating a particular set of content linguistic indicators. The study's findings provide a set of linguistic features that distinguish deceptive news types from those authentic types.

The results reveal that reported speech, passive voice, negation are the top shared features used in the two datasets, but these features are used less in real news articles. Moreover, authentic news uses proper nouns more than the other set. It corroborates the previous work of Newman et al. 2003 stated that self-references are less found in unreliable news (Newman et al., 2003). In contradiction, Rashkin et al. (2017) found that more first-person and second-person pronouns in less reliable or deceptive news (Rashkin et al., 2017). Ott and Rayson said that personal pronouns indicate creative writing (Ott et al., 2011; Rayson et al., 2001). Compared to dataset 2, reliable news tends to use less conjunction and to-infinitive attributes. It means that dataset 2 tends to be unreliable more than dataset 1 .

The results also display that unreliable news types use exaggerated words such as superlatives more than reliable ones, while comparatives are used more in truthful news reports. These findings are in accord with the conclusions by Ott et al. (2011), who stated the usage differences between superlative and comparative. Such differences demonstrate the substantial divergence in the content style of both news report types. As hypothesized, real sentences tend to be grammatical, while the same may not be the case for fake sentences (Badaskar et al., 2008). As noticed in earlier studies, most of the false headline's crafters employ excessive emotional adverbs to lure the readers or users to their content.

Untrusted sources tend to use hedging and vague words. Indeed, this is in line with the psychology theories (Burgoon et al., 2003) that declared deceivers show more "uncertainty and vagueness" and "indirect forms of expression." As noticed in Tables 1 and 2, reported speech is less likely to be used in trusted news reports type while it is one distinctive linguistic indicator of news reports in dataset 2. It indicates that dataset 2 tends to be unreliable more than reliable news. According to the study findings, fake news articles seem to use relatively more quotes than real articles. The results also showed a correlation between three features-reported speech, passive voice, negation, and repeatedly appearing in the real or fake language structure. Still, they are used more in fake news.

This paper stated that fake news detection is a real challenging task. Though many proposed approaches to check facts and detect news that identifies linguistic characteristics seem to be more effective and achieve reasonable and promising outcomes.

\section{Conclusion and Future Work}

This paper investigates fake news detection from a linguistic perspective, aiming to determine and predict the 
significant linguistic indicators used for news classifications and counterfeit content detection. This new perspective uses qualitative and quantitative analysis as a considerable and effective method that investigates and provides a computational representation of the content structure's discriminated linguistic features and style in textual data. More importantly, the study attempted to highlight the noticeable linguistic differences between authentic and fake news contents, thus reducing the blurry line between them.

In this study, the authors attempt to analyze two datasets linguistically. When comparing the linguistic characteristics of dataset 2 with those authentic texts download from the Politi-fact website, the results showed that dataset 2 tends to be fake rather than actual. Another exciting research line identifies a set of lexical-, grammatical- and syntactic features of fake news. The authors plan to investigate and explore more linguistic indicators for future work, specifically semantic and pragmatic related features.

\section{References}

Allcott, H., \& Gentzkow, M. (2017). Social media and fake news in the 2016 election. Journal of Economic Perspectives, 31(2), 211-236. https://doi.org/10.1257/jep.31.2.211

Badaskar, S., Agarwal, S., \& Arora, S. (2008). Identifying real or fake articles: Towards better language modeling. In Proceedings of the Third International Joint Conference on Natural Language Processing: Volume-II.

Balmas, M. (2014). When fake news becomes real: Combined exposure to multiple news sources and political attitudes of inefficacy, alienation, and cynicism. Communication Research, 41(3), 430-454. https://doi.org/10.1177/0093650212453600

Bessi, A., Scala, L., Rossi, Q., Zhang, W., \& Quattrociocchi, W. (2014). The economy of attention in the age of (mis)information. J. Trust Manage, 1, 12. https://doi.org/10.1140/epjst/e2015-50319-0

Bond, Jr, C. F., \& DePaulo, B. M. (2006). Accuracy of deception judgments. Personality and Social Psychology Review, 10(3), 214-234. https://doi.org/10.1207/s15327957pspr1003_2

Burfoot, C., \& Baldwin, T. (2009, August). Automatic satire detection: Are you having a laugh (pp. 161-164)? In Proceedings of the ACL-IJCNLP 2009 conference short papers. https://doi.org/10.3115/1667583.1667633

Burgoon, J. K., Blair, J. P., Qin, T., \& Nunamaker, J. F. (2003, June). Detecting deception through linguistic analysis (pp. 91-101). International Conference on Intelligence and Security Informatics. Springer, Berlin, Heidelberg. https://doi.org/10.1007/3-540-44853-5_7

Conroy, N. K., Rubin, V. L., \& Chen, Y. (2015). Automatic deception detection: Methods for finding fake news. Proceedings of the Association for Information Science and Technology, 52(1), 1-4. https://doi.org/10.1002/pra2.2015.145052010082

Golbeck, J., Mauriello, M., Auxier, B., Bhanushali, K. H., Bonk, C., Bouzaghrane, M. A., ... Falak, W. (2018, May). Fake news vs. satire: A dataset and analysis (pp. 17-21). In Proceedings of the 10th ACM Conference on Web Science. https://doi.org/10.1145/3201064.3201100

Levi, O., Hosseini, P., Diab, M., \& Broniatowski, D. A. (2019). Identifying Nuances in Fake News vs. Satire: Using Semantic and Linguistic Cues. arXiv preprint arXiv:1910.01160.

Markowitz, D. M., \& Hancock, J. T. (2014). Linguistic traces of a scientific fraud: The case of Diederik Stapel. PloS One, 9(8), e105937. https://doi.org/10.1371/journal.pone.0105937

Newman, M. L., Pennebaker, J. W., Berry, D. S., \& Richards, J. M. (2003). Lying words: Predicting deception from linguistic styles. Personality and Social Psychology Bulletin, 29(5), 665-675. https://doi.org/10.1177/0146167203029005010

Ott, M., Choi, Y., Cardie, C., \& Hancock, J. T. (2011). Finding deceptive opinion spam by any stretch of the imagination. arXiv preprint arXiv:1107.4557.

Qin, T., Burgoon, J. K., Blair, J. P., \& Nunamaker, J. F. (2005, January). Modality effects in deception detection and applications in automatic-deception-detection (pp. 23b-23b). In Proceedings of the 38th annual Hawaii international conference on system sciences. IEEE.

Rao, J. R., \& Rohatgi, P. (2000, August). Can pseudonymity really guarantee privacy (pp. 85-96)? In USENIX Security Symposium.

Rashkin, H., Choi, E., Jang, J. Y., Volkova, S., \& Choi, Y. (2017, September). Truth of varying shades: Analyzing 
language in fake news and political fact-checking (pp. 2931-2937). In Proceedings of the 2017 conference on empirical methods in natural language processing. https://doi.org/10.18653/v1/D17-1317

Rayson, P., Wilson, A., \& Leech, G. (2002). Grammatical word class variation within the British National Corpus sampler (pp. 295-306). In New Frontiers of Corpus Research. Brill Rodopi. https://doi.org/10.1163/9789004334113_020

Rubin, V. L., Conroy, N., Chen, Y., \& Cornwell, S. (2016, June). Fake news or truth? Using satirical cues to detect potentially misleading news (pp. 7-17). In Proceedings of the second workshop on computational approaches to deception detection. https://doi.org/10.18653/v1/W16-0802

Ruchansky, N., Seo, S., \& Liu, Y. (2017, November). Csi: A hybrid deep model for fake news detection (pp. 797-806). In Proceedings of the 2017 ACM on Conference on Information and Knowledge Management.

Shafqat, W., Lee, S., Malik, S., \& Kim, H. C. (2016, April). The language of deceivers: Linguistic features of crowdfunding scams (pp. 99-100). In Proceedings of the 25th International Conference Companion on World Wide Web. https://doi.org/10.1145/2872518.2889356

Shao, C., Ciampaglia, G. L., Flammini, A., \& Menczer, F. (2016, April). Hoaxy: A platform for tracking online misinformation (pp. 745-750). In Proceedings of the 25th international conference companion on world wide web. https://doi.org/10.1145/2872518.2890098

Shu, K., Sliva, A., Wang, S., Tang, J., \& Liu, H. (2017). Fake news detection on social media: A data mining $\begin{array}{lllll}\text { perspective. } & A C M & \text { SIGKDD } & \text { Explorations }\end{array}$ https://doi.org/10.1145/3137597.3137600

Shu, K., Suhang, W., \& Huan, L. (2017). Exploiting Tri-Relationship for Fake News Detection. ArXiv preprint arXiv:1712.07709.

Shu, K., Wang, S., \& Liu, H. (2019, January). Beyond news contents: The role of social context for fake news detection (pp. 312-320). In Proceedings of the Twelfth ACM International Conference on Web Search and Data Mining. https://doi.org/10.1145/3289600.3290994

Toma, C. L., \& Hancock, J. T. (2010, February). Reading between the lines: linguistic cues to deception in online dating profiles (pp. 5-8). In Proceedings of the 2010 ACM conference on Computer supported cooperative work. https://doi.org/10.1145/1718918.1718921

Undeutsch, U. (1967). Beurteilung der Glaubhaftigkeit von Aussagen (Evaluation of statement credibility). In Handbuch der Psychologie (Vol. 11: Forensische Psychologie, Undeutsch U. Hogrefe: G ottingen, pp. 126-181).

Warkentin, D., Woodworth, M., Hancock, J. T., \& Cormier, N. (2010, February). Warrants and deception in computer-mediated communication (pp. 9-12). In Proceedings of the 2010 ACM conference on Computer supported cooperative work. https://doi.org/10.1145/1718918.1718922

Zafarani, R., Zhou, X., Shu, K., \& Liu, H. (2019, July). Fake news research: Theories, detection strategies, and open problems (pp. 3207-3208). In Proceedings of the 25th ACM SIGKDD International Conference on Knowledge Discovery \& Data Mining. https://doi.org/10.1145/3292500.3332287

Zhang, L., \& Guan, Y. (2008, June). Detecting click fraud in pay-per-click streams of online advertising networks (pp. 77-84). In 2008 The 28th International Conference on Distributed Computing Systems. IEEE. https://doi.org/10.1109/ICDCS.2008.98

Zheng, R., Li, J., Chen, H., \& Huang, Z. (2006). A framework for authorship identification of online messages: Writing - style features and classification techniques. Journal of the American Society for Information Science and Technology, 57(3), 378-393. https://doi.org/10.1002/asi.20316

Zhou, X., Jain, A., Phoha, V. V., \& Zafarani, R. (2019). Fake news early detection: A theory-driven model. ArXiv preprint arXiv:1904.11679.

Zhou, X., Jain, A., Phoha, V. V., \& Zafarani, R. (2020). Fake news early detection: A theory-driven model. Digital Threats: Research and Practice, 1(2), 1-25. https://doi.org/10.1145/3377478

Zhou, X., \& Zafarani, R. (2018). Fake news: A survey of research, detection methods, and opportunities. arXiv preprint arXiv:1812.00315. 


\section{Copyrights}

Copyright for this article is retained by the author, with first publication rights granted to the journal.

This is an open-access article distributed under the terms and conditions of the Creative Commons Attribution license (http://creativecommons.org/licenses/by/4.0/). 